\title{
Convective Melting Modeling Approach for Phase Change Materials with Variable Boundary Heating
}

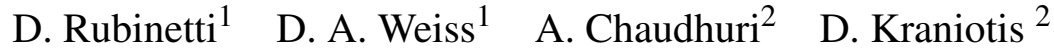 \\ ${ }^{1}$ Institute of Thermal- and Fluid-Engineering, University of Applied Sciences and Arts Northwestern Switzerland \\ ${ }^{2}$ Department of Civil Engineering and Energy Technology, OsloMet — Oslo Metropolitan University, Norway
}

\begin{abstract}
The present study conceives a numerical model for phase change materials following the apparent heat capacity method where the phase change occurs within a chosen temperature interval. A multiphysical modeling approach to satisfy the coupled momentum, energy and continuity conservation equations whilst avoiding numerical singularities is applied. By means of a 2D test-case geometry with variable boundary heating the influence of natural convection within the melted liquid zone is visualized. Corresponding non-dimensional governing equations are analysed to quantify the dominant contributing terms. It turns out that for sufficiently small Grashof number, or consequently small Rayleigh numbers the influence of natural convection can be neglected, thus simplyfing the problem substantially. The modeling approach has been adapted to a 2D-axisymmetric geometry within the scope of experimental validation. The simulation results and experimental data show reasonably good agreement.

The model is numerically stable and suitable to facilitate design of latent heat storage systems.
\end{abstract}

Keywords: Phase Change Materials, Fluid Flow, Heat Transfer, Phase Transition, Natural Convection, Melting, Latent Heat Storage

\section{Introduction}

Phase change material (PCM) finds an increasingly wide range of applications in thermal energy management due to their energy storage capabilities. The choice of a suitable PCM should satisfy physical, technical and economic requirements. In essence, these consist of materials with a characteristically large enthalpy of fusion in a temperature range smeared over a phase transition region. In order to appropriately design latent heat storage applications using PCMs, it is important to predict the complex behavior of the materials well enough. In the present study, a numerical model for implementation in COMSOL Multiphysics ${ }^{\mathrm{TM}}$ is conceived. Natural convection is accounted for by the Boussinesq approximation which allows the fluid to be treated as incompressible for inertial forces and compressible for buoyancy forces. The chosen material in this study is $n$-eicosane as specified in table 1, whose thermophysical properties come with great deal of experimental coverage (Muhammad et al., 2015;
Jones et al., 2006; Sparrow and Broadbent, 1982).

\section{Physical Model}

To model the physical nature of PCMs it is necessary to consider the disciplines of heat transfer and fluid flow. The strong coupling occurs due to natural convection and phase change effects constrained by the PCM container geometry as illustrated by figure 1 .

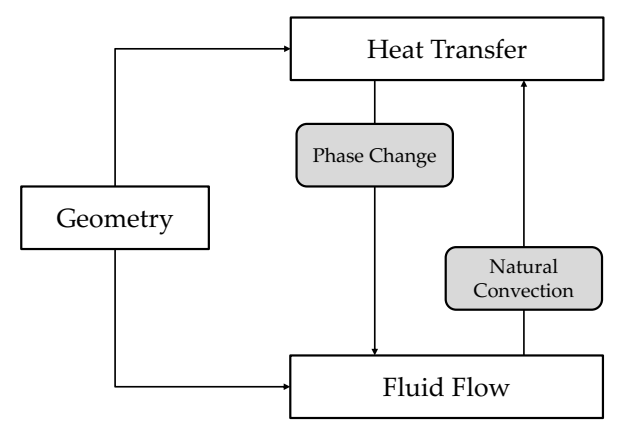

Figure 1. Coupled Physics.

The chosen geometry is a $2 \mathrm{D}$ square enclosure with 1 $\mathrm{cm}$ side-length $(H)$. Figure 2 shows the computational setup. On the right wall a Dirichlet boundary condition for three different temperatures is applied: $T_{R}=40 / 50 / 70^{\circ} \mathrm{C}$. The three remaining boundaries represent thermal insulation. Boundaries are also prescribed as no-slip walls wherever applicable.

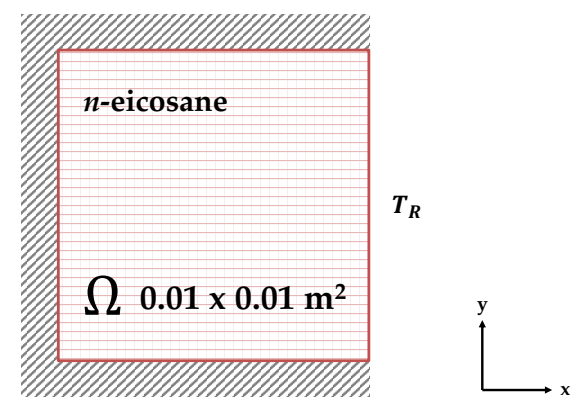

Figure 2. Geometry of the 2D model.

The melting process occurs within a time-range of $0<t<1000 \mathrm{~s}$ for all temperatures. 


\section{NOMENCLATURE}

\begin{tabular}{|c|c|c|}
\hline$A_{m}$ & mushy zone coefficient & $\mathrm{kg} \mathrm{m}^{-3} \mathrm{~s}^{-1}$ \\
\hline$C_{p}$ & $\begin{array}{l}\text { specific heat capacity at con- } \\
\text { stant pressure }\end{array}$ & $\mathrm{J} \mathrm{kg}^{-1} \mathrm{~K}^{-1}$ \\
\hline$D$ & Gaussian distribution & - \\
\hline$F$ & volumetric force on fluid & $\mathrm{Nm}^{-3}$ \\
\hline$g$ & gravitational acceleration & $\mathrm{ms}^{-2}$ \\
\hline$H$ & domain length & $\mathrm{m}$ \\
\hline$k$ & thermal conductivity & $\mathrm{W} \mathrm{m}^{-1} \mathrm{~K}^{-1}$ \\
\hline$L$ & latent heat / heat of fusion & $\mathrm{J} \mathrm{kg}^{-1}$ \\
\hline$p$ & pressure & $\mathrm{Pa}$ \\
\hline$R$ & radius & $\mathrm{m}$ \\
\hline$r$ & radial coordinate & $\mathrm{m}$ \\
\hline$T$ & temperature & $\mathrm{K}$ \\
\hline$\Delta T$ & $\begin{array}{l}\text { phase transition temperature } \\
\text { range }\end{array}$ & K \\
\hline$T_{m}$ & melting temperature & $\mathrm{K}$ \\
\hline$T_{R}$ & wall temperature & $\mathrm{K}$ \\
\hline$t$ & time & $\mathrm{s}$ \\
\hline$u$ & velocity & $\mathrm{ms}^{-1}$ \\
\hline$u_{0}$ & characteristic velocity & $\mathrm{ms}^{-1}$ \\
\hline$x$ & $\mathrm{x}$-coordinate & $\mathrm{m}$ \\
\hline$y$ & $\mathrm{y}$-coordinate & $\mathrm{m}$ \\
\hline$z$ & $\mathrm{z}$-coordinate & $\mathrm{m}$ \\
\hline$\alpha$ & thermal diffusivity & $\mathrm{m}^{2} \mathrm{~s}^{-1}$ \\
\hline$\beta$ & thermal expansion coefficient & $\mathrm{K}^{-1}$ \\
\hline$\varepsilon$ & Carman-Kozeny constant & - \\
\hline$\mu$ & dynamic viscosity & Pas \\
\hline$v$ & kinematic viscosity & $\mathrm{m}^{2} \mathrm{~s}^{-1}$ \\
\hline$\Phi_{v}$ & dissipation function & $\mathrm{s}^{-2}$ \\
\hline$\rho$ & density & $\mathrm{kg} \mathrm{m}^{-3}$ \\
\hline$\theta$ & melt fraction & - \\
\hline $\mathrm{Br}$ & Brinkman number & - \\
\hline Gr & Grashof number & - \\
\hline $\operatorname{Pr}$ & Prandtl number & - \\
\hline $\mathrm{Ra}$ & Rayleigh number & - \\
\hline $\operatorname{Re}$ & Reynolds number & - \\
\hline \multicolumn{3}{|c|}{ Super- and subscripts } \\
\hline$l$ & liquid & \\
\hline ref & reference quantity & \\
\hline$s$ & solid & \\
\hline$\sim$ & dimensionless quantity & \\
\hline
\end{tabular}

Table 1. Properties of $n$-eicosane

\begin{tabular}{lcc}
\hline & Solid & Liquid \\
\hline$\rho$ & 910 & 769 \\
$k$ & 0.423 & 0.146 \\
$C_{p}$ & 1926 & 2400 \\
$\beta$ & - & $8.161 \times 10^{-4}$ \\
$T_{m}$ & 36.4 & - \\
$L$ & 248000 & - \\
\hline
\end{tabular}

\section{Numerical Model}

Modeling of phase change phenomena can be achieved by splitting the computation domain $\Omega$ into a subdomain for the solid fraction and a subdomain for the liquid fraction. A major numerical drawback hereof is the difficulty of tracking the solid/liquid interface which require delicate adaptive meshing methods to guarantee both convergence and reasonable representation of the physical processes (Lewis et al., 2004). The predominant state-of-art approach for the modeling of phase change materials is based on enthalphy methods, i.e. the enthalphy-porosity formulation (Dutil et al., 2011). The enthalphy-porosity formulation requires only one mathematical model. By means of temperature-dependent material laws the material is considered both as solid and liquid while the phase transition region is denoted as mushy region.

\subsection{Material description}

The enthalpy-porosity method bases all material properties such as melt fraction, heat capacity, density and thermal conductivity to the current temperature $T$ of the material. The enthalpy-porosity formulation quantifies the melt fraction $\theta(T)$ in terms of a temperature-dependent ramp function from 0 to 1 over a chosen phase transition temperature interval $\Delta T$. It is centered around the melting threshold temperature $T_{m}$ (Kheirabadi and Groulx, 2015) as,

$$
\theta(T)= \begin{cases}0, & \text { for } T<T_{m}-\Delta T / 2 \\ \frac{T-\left(T_{m}-\Delta T / 2\right)}{\Delta T}, & \text { for } T_{m}-\Delta T / 2<T<T_{m}+\Delta T / 2 \\ 1, & \text { for } T>T_{m}+\Delta T / 2\end{cases}
$$

A graphical representation of the melt fraction function is shown in figure 3a. To account for the contribution of latent heat over the phase transition region, a Gaussian distribution function $D(T)$ ensures energy conservation over the chosen temperature interval as illustrated in figure $3 \mathrm{~b}$. $D(T)$ is given by,

$$
D(T)=\frac{e^{-\frac{\left(T-T_{m}\right)^{2}}{(\Delta T / 4)^{2}}}}{\sqrt{\pi(\Delta T / 4)^{2}}} .
$$

The definitions according to equation (1) and (2) allow the modification of the heat capacity $C_{p}$ of the PCM as,

$$
C_{p}(T)=C_{p, s}+\theta(T)\left(C_{p, l}-C_{p, s}\right)+D(T) L .
$$

Note that, the heat capacity increases notably in the mushy region due to the latent heat contribution as seen in figure $3 \mathrm{c}$. Temperature-dependent thermal conductivity and density are defined analogously like the heat capacity without latent heat contribution term.

In numerical point of view, the choice of the temperature transition range $\Delta T$ requires careful consideration. The 
sharp gradients of the modified heat capacity may lead to convergence issues if $\Delta T$ is small (i.e. $\sim 0.1 \mathrm{~K}$ ) and the mesh resolution at the interface does not cover the imposed range. A small $\Delta T$ means greater accuracy coming with greater computational effort. A qualitative initial choice in the range of $5 \mathrm{~K}$ may be used (Murray and Groulx, 2011). A reasonable trade-off between accuracy and computational effort in the test-cases of the present study is the chosen value of $2 \mathrm{~K}$. The work is carried out with the commercial tool COMSOL Multiphysics ${ }^{\mathrm{TM}}$ which offers a suitable user-input environment for implementing the enthalpy-porosity method.

\subsection{Governing Equations}

The governing equations to describe the physics include the laminar Navier-Stokes equations, the continuity equation and the energy conservation equation. Here the set of equations for a successful implementation in COMSOL Multiphysics $^{\mathrm{TM}}$ is presented.

\subsubsection{Continuity equation}

The continuity equation assuming constant density is defined as follows

$$
\nabla \cdot \boldsymbol{u}=0
$$

\subsubsection{Momentum equation}

The incompressible momentum equation assumes constant density yielding

$$
\rho \frac{\partial \boldsymbol{u}}{\partial t}+\rho(\boldsymbol{u} \cdot \nabla) \boldsymbol{u}=-\nabla p+\nabla \cdot(\mu(T) \nabla \boldsymbol{u})+S(T) \boldsymbol{u}+\boldsymbol{F}
$$

where $S(T) \boldsymbol{u}$ is an additional modeling term to account for the flow within the mushy zone. Its component $S(T)$ is described by the Carman-Kozeny equation as

$$
S(T)=A_{m} \frac{(1-\theta(T))^{2}}{\theta(T)^{3}+\varepsilon},
$$

with the modeling constants $A_{m}=10^{6} \mathrm{~kg} \mathrm{~m}^{-3} \mathrm{~s}^{-1}$ and $\varepsilon=10^{-3}$ (Samara et al., 2012; Kheirabadi and Groulx, 2015). The function $S(T)$ forms a sink-term in the momentum equation. For the solid fraction, the momentum equation (5) shall return the trivial $\boldsymbol{u}=0$ solution to ensure immobility. As illustrated in figure $3 \mathrm{~d}$, the $S(T)$ affects the solid fraction as well as the solid-dominated part of the mushy zone $\left(T<T_{m}\right)$. The modeling term $S(T)$ overrides every other terms in the momentum equation in the solid region. To achieve convergence in the model, however, it is recommended to additionally modify the viscosity (Kheirabadi and Groulx, 2015) in COMSOL Multiphysics $^{\mathrm{TM}}$ as below

$$
\mu(T)=\mu_{l}(1+S(T)),
$$

with the empirical viscosity-temperature relation (Muhammad et al., 2015) for $n$-eicosane,

$$
\mu_{l}=\left(9 \times 10^{-4} T^{2}-0.6529 T+119.94\right) \times 10^{-3} .
$$

where $T$ is given in $[\mathrm{K}]$ and the dynamic viscosity $\mu_{l}$ in [Pa s]. Consequently the dissipation term in the momentum equation overrides the other terms within the solid regime. That way it is guaranteed that flow is induced only in the liquid fraction within the computational domain despite of solving the momentum equation for both solid and liquid fraction.

The force term accounting for gravity and natural convection is given by the Boussinesq approximation. This is implemented as (Bird et al., 2007),

$$
\boldsymbol{F}=\rho_{l}\left(1-\beta\left(T-T_{m}\right)\right) \boldsymbol{g} .
$$

\subsubsection{Energy equation}

The energy equation in terms of the temperature distribution $\mathrm{T}$ is defined by

$$
\rho(T) C_{p}(T) \frac{\partial T}{\partial t}+\rho(T) C_{p}(T) \boldsymbol{u} \cdot \nabla T=\nabla \cdot(k(T) \nabla T) .
$$

\subsection{Mesh}

The mesh for the test-case geometry is a mapped mesh with 40000 quadrilateral elements. The mesh is strategically refined towards the heated right boundary and on the top boundary to tackle potential complications with noslip walls during early stages of melting when the velocitites within the fluid fraction are close to zero.

On a high-performance cluster with $16 \mathrm{CPUs}$ and 94 GB RAM the computational runtime amounts to approximately 7.5 hours for a simulation time of $t=1000 \mathrm{~s}$. To ensure convergence it is recommended to use a fully coupled direct solver configured for the Newton nonlinear method.

\section{Results}

The results for all test cases are summarized in figure 4 . It appears that the melt fraction has a strong dependence on temperature, indicating that the natural convection plays an important role. To quantify the importance of natural convection the non-dimensional forms of the governing equations for non-isothermal flow are investigated. A temperature-independent liquid viscosity of $\mu_{l}=0.008$ Pas (Kheirabadi and Groulx, 2015) in the simulation and the following non-dimensional variables are used (Bird et al., 2007)

$$
\begin{aligned}
& \tilde{x}=\frac{x}{H} \\
& \tilde{y}=\frac{y}{H} \\
& \tilde{p}=\frac{p-p_{r e f}}{\rho u_{0}^{2}} \\
& \tilde{t}=\frac{u_{0} t}{H} \\
& \tilde{\boldsymbol{u}}=\frac{\boldsymbol{u}}{u_{0}} \\
& \tilde{T}=\frac{T-T_{r e f}}{T_{R}-T_{r e f}} \\
& \tilde{\Phi}_{v}=\left(\frac{H}{u_{0}}\right)^{2} \Phi_{v} \quad \tilde{\nabla}=H \nabla \quad \frac{D}{D \tilde{t}}=\left(\frac{H}{u_{0}}\right) \frac{D}{D t} .
\end{aligned}
$$



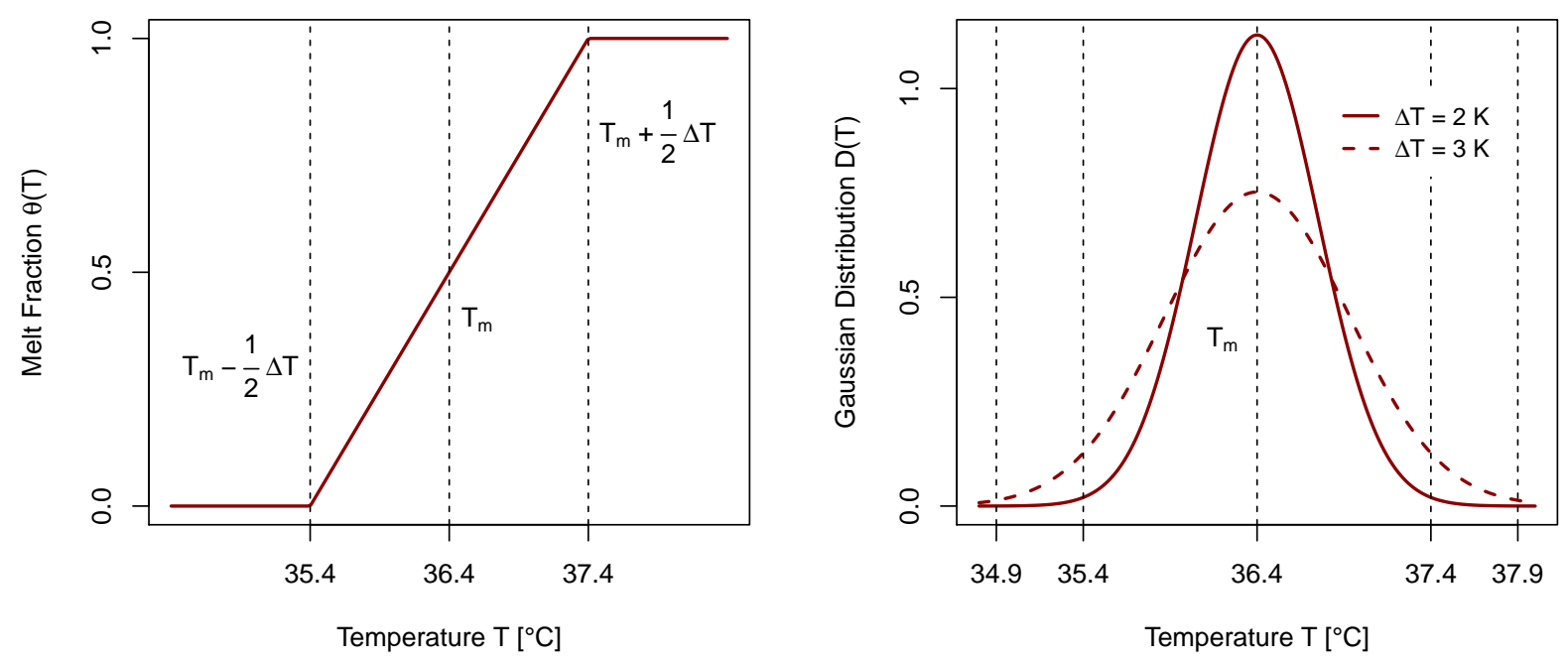

(a) Melt fraction function $\theta(T)$, implemented as piecewise (b) Gaussian distribution function $D(T)$, shown for different function.

$\Delta T \mathrm{~s}$.
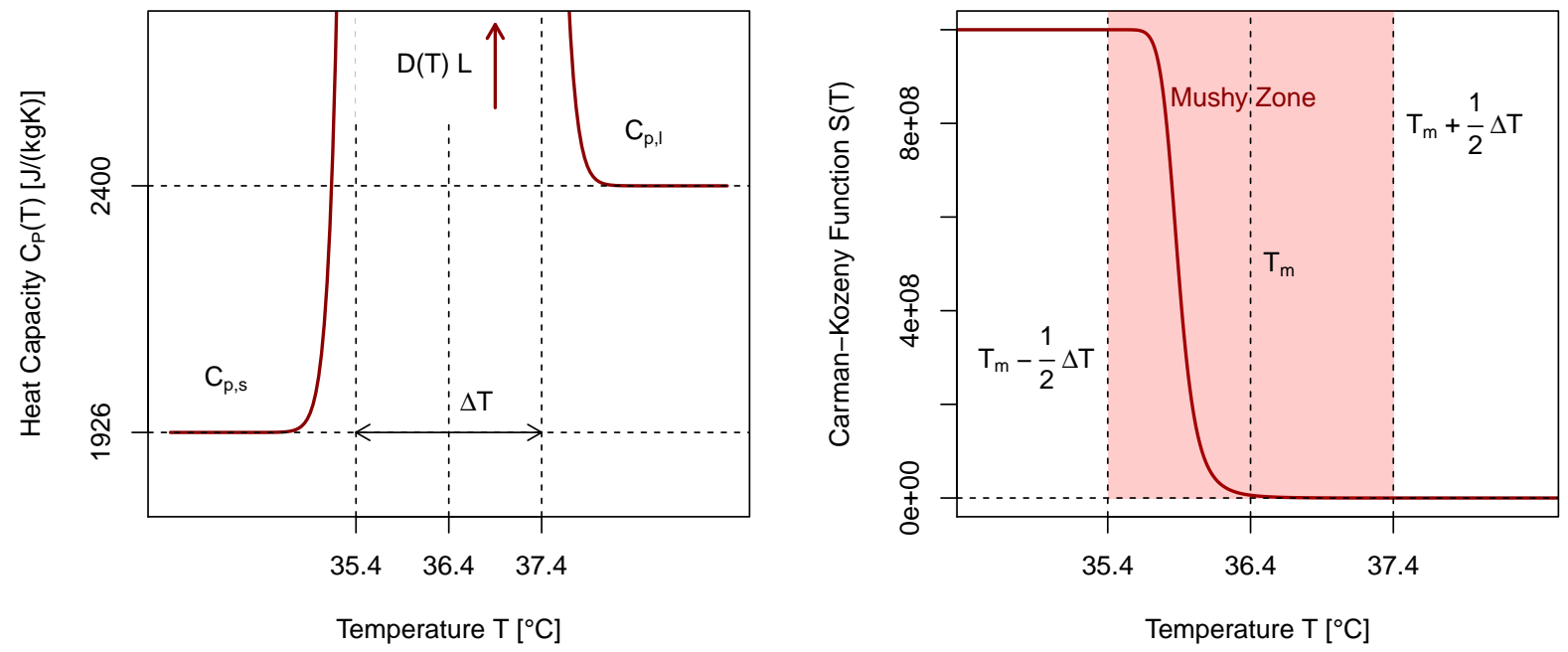

(c) Heat capacity function $C_{p}(T)$, rising up to a maximum (d) Carman-Kozeny function to model the sink-term and visvalue of $D(T) L=272000 \mathrm{~J} \mathrm{~kg}^{-1} \mathrm{~K}^{-1}$. cosity of the solid fraction.

Figure 3. Temperature-dependent modeling functions as presented by (Kheirabadi and Groulx, 2015) for the chosen PCM $n$ eicosane. 
with $u_{0}=v / H$ being the characteristic velocity. Thus the continuity equation yields

$$
\tilde{\nabla} \cdot \tilde{\boldsymbol{u}}=0 .
$$

The momentum equation is expressed in terms of the total derivative and contains two dimensionless groups

$$
\begin{aligned}
\frac{D \tilde{\boldsymbol{u}}}{D \tilde{t}}= & -\tilde{\nabla} \tilde{p}+\left[\frac{\mu}{u_{0} \rho H}\right] \tilde{\nabla}^{2} \tilde{\boldsymbol{u}} \\
& -\left[\frac{g \beta\left(T_{R}-T_{m}\right) H}{u_{0}^{2}}\right]\left(\frac{\boldsymbol{g}}{g}\right)\left(\tilde{T}-\tilde{T}_{m}\right),
\end{aligned}
$$

whereas the energy equation features a dissipation term and writes

$$
\frac{D \tilde{T}}{D \tilde{t}}=\left[\frac{k}{u_{0} \rho H C_{p}}\right] \tilde{\nabla}^{2} \tilde{T}+\left[\frac{\mu u_{0}}{\rho H C_{p}\left(T_{R}-T_{m}\right)}\right] \tilde{\Phi}_{v} .
$$

The values of the four dimensionless groups for the present test-case are summarized in table 2 with the following relevant dimensionless numbers

$$
\begin{array}{ll}
\text { Brinkman } & \mathrm{Br}=\frac{\mu u_{0}{ }^{2}}{k \Delta T}, \\
\text { Grashof } & \mathrm{Gr}=\frac{g \beta \Delta T H^{3}}{v^{2}}, \\
\text { Prandtl } & \operatorname{Pr}=\frac{v}{\alpha}, \\
\text { Rayleigh } & \mathrm{Ra}=\frac{g \beta \Delta T H^{3}}{\alpha \nu}=\mathrm{GrPr}, \\
\text { Reynolds } & \mathrm{Re}=\frac{\rho u_{0} H}{\mu} .
\end{array}
$$

As can be seen from the evaluation the sharpest increase with temperature is the buoyancy term in the momentum equation. Comparing with the simulation results in figure 4 , it is safe to assume that natural convection can be neglected for $\mathrm{Gr} / \mathrm{Re}^{2}<266$ which reduces the model complexity substantially as the energy equation alone is sufficient to investigate the phase change without CFD coupling.

\subsection{Validation}

The implemented model is validated using experimental data of (Jones et al., 2006). Their experimental setup with $n$-eicosane in a cylindrical enclosure as illustrated in figure 5 with the wall temperature $T_{R}=45^{\circ} \mathrm{C}$ and simulation time $t=18000 \mathrm{~s}$. Additionally we set a subcooling wall at the bottom with $T_{B}=32{ }^{\circ} \mathrm{C}$ with the effect that a part of the enclosure shall remain solid according to (Jones et al., 2006).

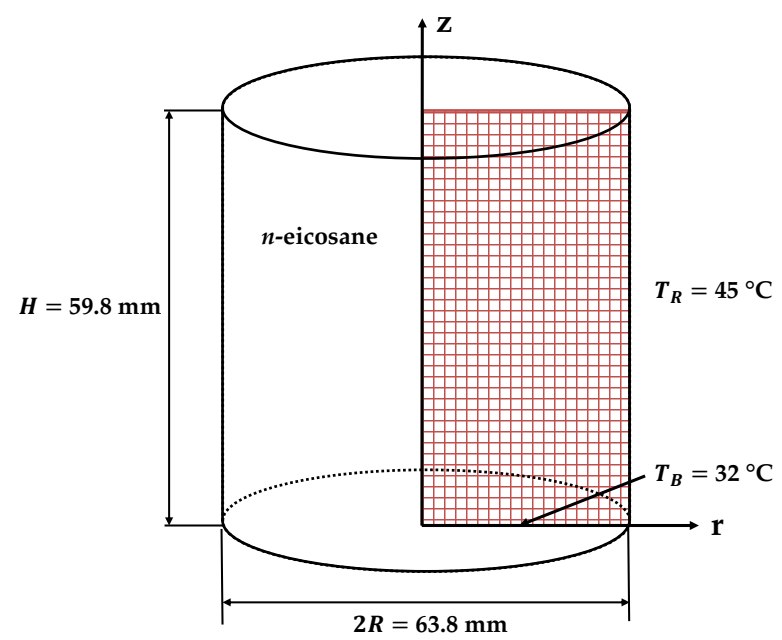

Figure 5. 2D axisymmetric geometry for experimental validation

The computational cost on the same high-performance cluster increases for the validation case to approximately 25 hours.

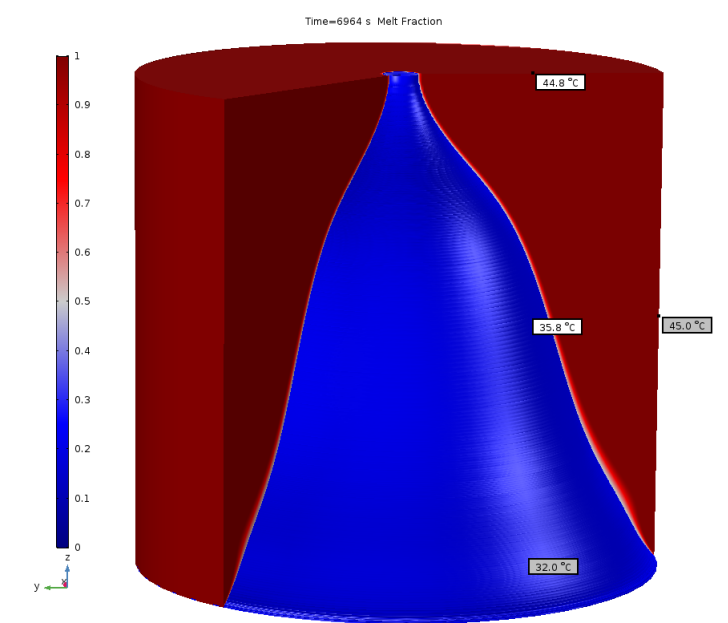

Figure 6. Melt fraction at $t=6964 \mathrm{~s}$ with temperature labels.

The evolution of the melting front show reasonably good agreement with the experimental results as shown in figure 7.

\subsection{Discussion}

The dimensionless estimation of the terms as indicated in table 2 can be of useful guidance when setting up a phase change related model. In fact, for low Gr and Ra numbers, the model might deliver acceptable results solving the energy equation only. Low $\mathrm{Gr}$ and $\mathrm{Ra}$ numbers are achieved when the temperature difference $T_{R}-T_{m}$ has approximately the same order of magnitude as the phase transition temperature range $\Delta T$. The analysis with dimensionless numbers presented here targets the liquid fraction only. Within the mushy zone a different behavior can be expected, i.e. the dissipation term in the energy equation will override the other terms due to a sharp increase in vis- 
Table 2. Dimensionless groups in non-isothermal flow equations

\begin{tabular}{|c|c|c|c|}
\hline \multirow[t]{2}{*}{ Dimensionless group } & \multicolumn{3}{|c|}{$T_{R}$} \\
\hline & $40^{\circ} \mathrm{C}$ & $55^{\circ} \mathrm{C}$ & $70^{\circ} \mathrm{C}$ \\
\hline$\left[\frac{\mu}{u_{0} \rho H}\right]=\frac{1}{\operatorname{Re}}$ & 1 & 1 & 1 \\
\hline$\left[\frac{g \beta\left(T_{R}-T_{m}\right) H}{u_{0}^{2}}\right]=\frac{\mathrm{Gr}}{\operatorname{Re}^{2}}=\frac{\mathrm{Ra}}{\operatorname{PrRe}^{2}}$ & 266 & 1376 & 2486 \\
\hline$\left[\frac{k}{u_{0} \rho H C_{p}}\right]=\frac{1}{\operatorname{RePr}}$ & 0.008 & 0.008 & 0.008 \\
\hline$\left[\frac{\mu u_{0}}{\rho H C_{p}\left(T_{R}-T_{m}\right)}\right]=\frac{\mathrm{Br}}{\operatorname{RePr}}$ & $1.25 \times 10^{-10}$ & $2.42 \times 10^{-11}$ & $1.34 \times 10^{-11}$ \\
\hline
\end{tabular}

cosity due to the Carman-Kozeny porosity function $S(T)$ as seen in equation (7).

Nonetheless, the modeling approach can seamlessly be adapted to other materials. The validation case shows reasonably good agreement between simulation and experimental results. The deviations can be explained by uncertainties in the retrieval of experimental data (Jones et al., 2006) and in the choice of modeling parameters, e.g. $\Delta T$. Smearing out the phase transition over a temperature range $\Delta T$ is questionable in terms of physical accuracy as its purpose and necessity is the handling of numerical singularities. Further points to consider for model improvement include

- mesh independence study

- quantification of viscous dissipation in the mushy zone

- volumetric expansion during melting

- aging of the material.

\section{Conclusion}

This work provides a modeling guideline for computing phase change phenomena on a stationary grid, coupling heat transfer and fluid dynamics. From the results it can be seen that the increasing influence of natural convection also increases to the amount of molten material. While the dimensionless estimation delivers reliable reference values for an estimation, the simulation provides visuals to spot further design improvements. Of particular interest are the local (grid) Gr resp. Ra numbers which indicate the exact region of influence within the geometry.

The presented model for the implementation of phase change phenomena in COMSOL Multiphysics ${ }^{\mathrm{TM}}$ is numerically stable and physically accurate enough to facilitate the design of latent heat storage systems.

\section{References}

Byron R. Bird, Warren E. Stewart, and Edwin N. Lightfoot. Transport Phenomena. Wiley, New York, second edition edition, 2007.

Yvan Dutil, Daniel R. Rousse, Nizar Ben Salah, Stéphane Lassue, and Laurent Zalewski. A review on phase-change materials: mathematical modeling and simulations. Renewable and sustainable Energy reviews, 15(1):112-130, 2011. doi:10.1016/j.rser.2010.06.011.

Benjamin J. Jones, Dawei Sun, Shankar Krishnan, and Suresh V. Garimella. Experimental and numerical study of melting in a cylinder. International Journal of Heat and Mass Transfer, 49(15-16):2724-2738, 2006.

Ali C. Kheirabadi and Dominic Groulx. Simulating phase change heat transfer using comsol and fluent: Effect of the mushy-zone constant. Computational Thermal Sciences: An International Journal, 7(5-6), 2015. doi:10.1615/ComputThermalScien.2016014279.

Roland W. Lewis, Perumal Nithiarasu, and Kankanhalli N. Seetharamu. Fundamentals of the finite element method for heat and fluid flow. John Wiley and Sons, 2004.

M.D. Muhammad, O. Badr, and H. Yeung. Validation of a cfd melting and solidification model for phase change in vertical cylinders. Numerical Heat Transfer, Part A: Applications, 68 (5):501-511, 2015. doi:10.1080/10407782.2014.994432.

Robynne E. Murray and Dominic Groulx. Modeling convection during melting of a phase change material. In Proceedings of the COMSOL Conference, 2011.

Farid Samara, Dominic Groulx, and Pascal H. Biwole. Natural convection driven melting of phase change material: comparison of two methods. In Excerpt from the Proceeding of the COMSOL Conference, 2012.

E.M. Sparrow and J.A. Broadbent. Inward melting in a vertical tube which allows free expansion of the phase-change medium. Journal of Heat Transfer, 104(2):309-315, 1982. doi:10.1115/1.3245089. 


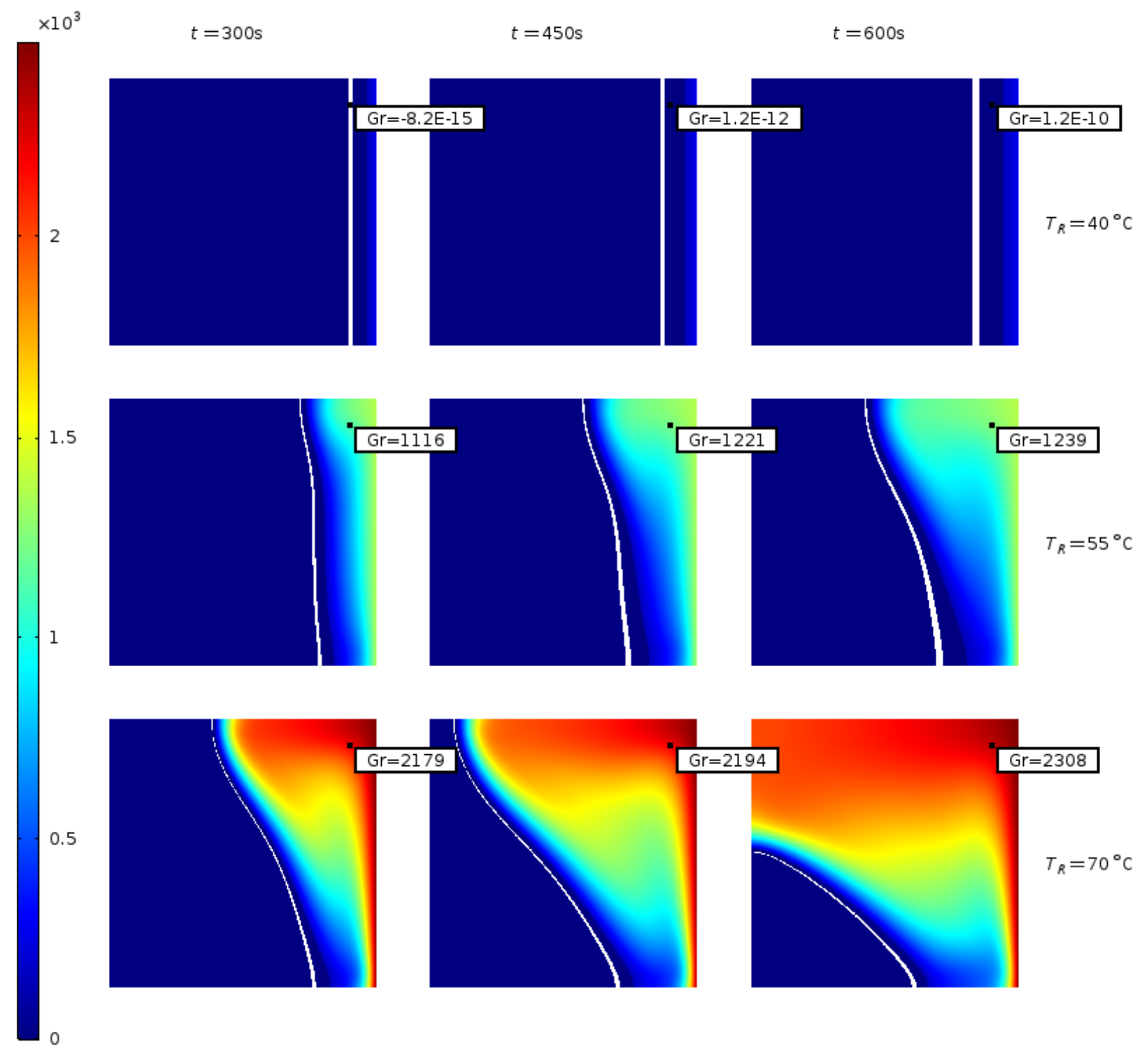

Figure 4. Simulation result of the grid Grashof number with white isoline tracking the melting front. As expected from the computed values in table 2 , Gr increases strongly with temperature. Due to the constant viscosity $\mu_{l}$ and thermal diffusivity $\alpha$ the Gr number is directly linked to Rayleigh number by a scaling factor of $1 / \operatorname{Pr}=\alpha \rho_{l} / \mu_{l}$. Thus, values of Gr can be used as reference to measure the intensity of buoyancy resp. driving force of natural convection. The simulation results visualize the main regions of influence of the convective flow; for $T_{R}=40{ }^{\circ} \mathrm{C}$ the front moves parallel away from the heated boundary insinuating no contribution from the momentum equation. On the other extreme, the $T_{R}=70{ }^{\circ} \mathrm{C}$ case clearly shows how the phase interface bends under influence of convection. 


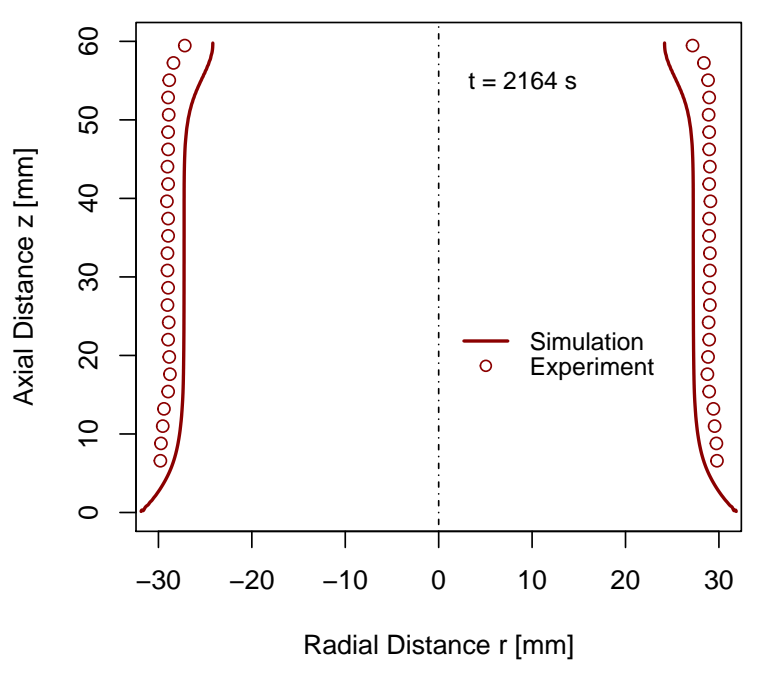

(a) Interface location at $t=2164 \mathrm{~s}$.

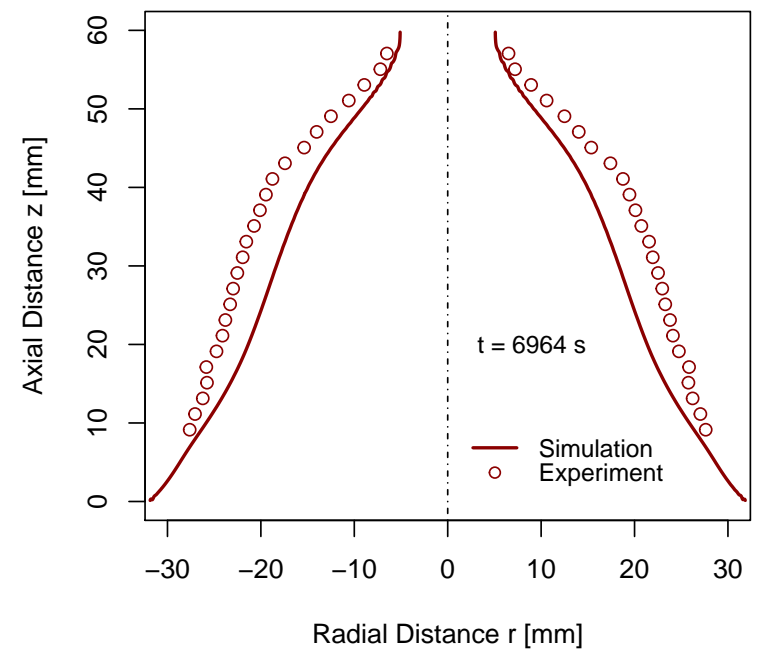

(b) Interface location at $t=6964 \mathrm{~s}$.

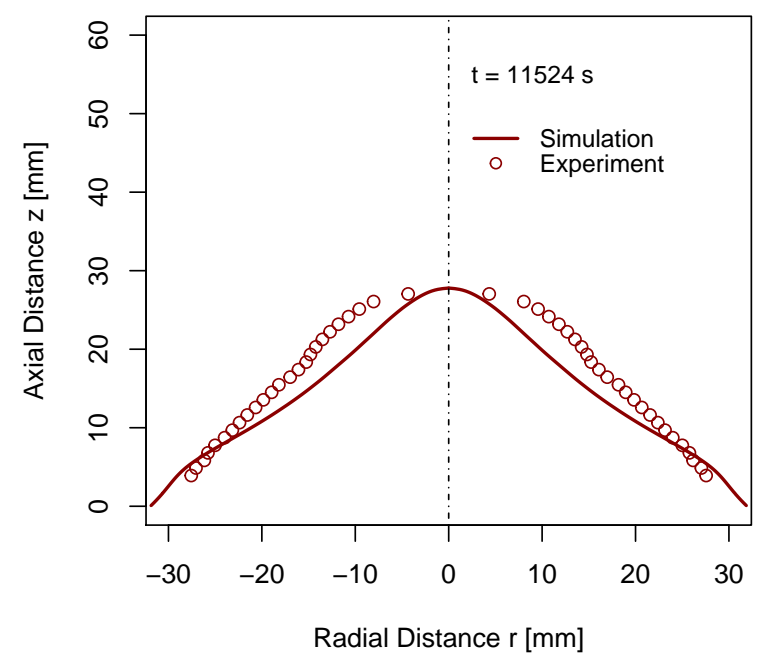

(c) Interface location at $t=11524 \mathrm{~s}$.

Figure 7. Comparison of simulation results with experimental data (Jones et al., 2006). 